\title{
A novel decomposition of aggregate total factor productivity change
}

\author{
Bert M. Balk ${ }^{1}{ }^{1}$ \\ Published online: 14 April 2019 \\ (c) The Author(s) 2019
}

\begin{abstract}
An industry is an ensemble of individual firms (decision making units) which may or may not interact with each other. Similarly, an economy is an ensemble of industries. In National Accounts terms this is symbolized by the fact that the nominal value added produced by an industry or an economy is the simple sum of firm-, or industry-specific nominal value added. From this viewpoint it is natural to expect that there is a relation between (aggregate) industry or economy productivity and the (disaggregate) firm- or industry-specific productivities. In an earlier paper (Statistica Neerlandica 2015) three time-symmetric decompositions of aggregate value-added-based total factor productivity change were developed. In the present paper a fourth decomposition will be developed. A notable difference with the earlier paper is that the development is cast in terms of levels rather than indices. Various aspects of this new decomposition will be discussed and links with decompositions found in the literature unveiled. It turns out that one can dispense with the usual neo-classical assumptions.
\end{abstract}

Keywords Productivity $\cdot$ Aggregation $\cdot$ Decomposition $\cdot$ Domar weight $\cdot$ Index number theory

JEL codes $\mathrm{C} 43 \cdot \mathrm{D} 24 \cdot \mathrm{O} 47$

\section{Introduction}

This introduction ${ }^{1}$ sketches the context. The first article of this series, Balk (2010), considered productivity measurement for a single, consolidated production unit. In terms of levels, productivity is defined as real output divided by real input. Real output or input means nominal output or input deflated by some output- or input-specific price index, respectively. For the production unit considered, productivity change (through time) can then be measured as a

Earlier versions of this paper have been presented at the XV European Workshop on Efficiency and Productivity Analysis, London, 13-15 June 2017 and the 12th Asia-Pacific Productivity Conference, Seoul, 4-6 July 2018.

Bert M. Balk

bbalk@rsm.nl

1 Rotterdam School of Management, Erasmus University, Rotterdam, The Netherlands

\footnotetext{
${ }^{1}$ Adapted from the corresponding section of Balk (2018a).
}

difference or a ratio of productivities. In the latter case it appears that productivity change can also be defined directly as output quantity index divided by input quantity index.

The choice of the output and input concepts appears to be critical. Three main models can be distinguished: KLEMS$\mathrm{Y}, \mathrm{KL}-\mathrm{VA}$, and K-CF. Taking the composition of capital input cost into account, as set out in the companion paper Balk (2011), two more models can be added, namely KLNVA and K-NCF. Assuming profit (defined as revenue minus total cost) to be equal to zero, or, what amounts to the same, replacing an exogenous interest rate by an endogenous rate, multiplies the number of models by two. And the introduction of a capital utilization rate further complicates the picture. Thus, there is a lot of choice here, with not unimportant empirical consequences, as illustrated by Vancauteren et al. (2012).

Production units exist at various levels of aggregation. We see plants, enterprises, industries, countries, to name just some types of production units materializing in analyses of productivity change. Usually such units appear, more or less naturally, arranged into higher level aggregates. For instance, a number of plants belonging to the same 
enterprise; a certain type of enterprises defining an industry; a number of industries defining the 'measurable' part of a national economy; national economies making up the world economy. It is not difficult to perceive several sorts of hierarchy here.

As in any of these situations the structure is the samethere is an ensemble of production units, and the ensemble itself may or may not be considered as a higher level production unit -, it is interesting to study the relation between aggregate productivity (change) and productivity (change) of the aggregate.

There are basically two approaches here. Balk (2016) reviews and discusses the so-called bottom-up approach, the approach that takes an ensemble of individual production units as the fundamental frame of reference. The top-down approach is the subject of three other papers, namely Balk (2014) plus Dumagan and Balk (2016) on labour productivity, and Balk (2015) on total factor productivity. The connection between the two approaches is considered in Balk (2018a).

The present paper basically continues Balk (2015). In the 2015 paper three (time-) symmetric decompositions of aggregate value-added based total factor productivity change were developed. In the present paper a fourth decomposition will be developed. A notable difference with the earlier paper is that the development is cast in terms of levels rather than indices.

This paper unfolds as follows. Section 2 refreshes the accounting framework; nothing new there. Value-added based total factor productivity is defined as real value added divided by real primary input; hence, Section 3 defines these two concepts. Section 4 shows that aggregate valueadded based total factor productivity change essentially consists of three components: a weighted mean of individual value-added based total factor productivity changes, a factor reflecting reallocation between the production units, and a factor reflecting relative price changes at the input and output sides. Section 5 shows how the reallocation factor can be decomposed further into the contributions of the separate primary inputs. Section 6 shows how the decomposition derived in Section 4 changes if value-added based productivity change is replaced by gross-output based productivity change. Section 7 contains a key result: under mild restrictions on the relation between aggregate and individual deflators, if profit equals 0 then the reallocation factor vanishes, and aggregate value-added based total factor productivity change equals the product of Domarweighted individual gross-output based total factor productivity changes. In Section 8 we take a further step by assuming that the production units share the same timeinvariant production function. We then obtain a decomposition in terms of technical efficiency change, scale and mix effects.

\section{Accounting framework}

We consider ${ }^{2}$ a (static) ensemble (or set) $\mathcal{K}$ of consolidated production units ${ }^{3}$, operating during a certain time period $t$ in a certain country or region. For each unit the KLEMS-Y $e x$ post accounting identity in nominal values (or, in current prices) reads

$$
C_{K L}^{k t}+C_{E M S}^{k t}+\Pi^{k t}=R^{k t}(k \in \mathcal{K}),
$$

where $C_{K L}^{k t}$ denotes the primary input cost, $C_{E M S}^{k t}$ the intermediate inputs cost, $R^{k t}$ the revenue, and $\Pi^{k t}$ the profit (defined as remainder). Intermediate inputs cost (on energy, materials, and business services) and revenue concern generally tradeable commodities. It is presupposed that there is some agreed-on commodity classification, such that $C_{E M S}^{k t}$ and $R^{k t}$ can be written as sums of quantities times (unit) prices of these commodities. Of course, for any production unit most of these quantities will be zero. It is also presupposed that output prices are available from a market or else can be imputed. Taxes on production are supposed to be allocated to the $K$ and $L$ classes.

The commodities in the capital class $K$ concern owned tangible and intangible assets, organized according to industry, type, and age class. Each production unit uses certain quantities of those assets, and the configuration of assets used is in general unique for the unit. Thus, again, for any production unit most of the asset cells are empty. Prices are defined as unit user costs and, hence, capital input cost $C_{L}^{k t}$ is a sum of prices times quantities.

Finally, the commodities in the labour class $L$ concern detailed types of labour. Though any production unit employs specific persons with certain capabilities, it is usually their hours of work that count. Corresponding prices are hourly wages. Like the capital assets, the persons employed by a certain production unit are unique for that unit. It is presupposed that, wherever necessary, imputations have been made for self-employed workers. Henceforth, labour input cost $C_{L}^{k t}$ is a sum of prices times quantities.

Total primary input cost is the sum of capital and labour input cost, $C_{K L}^{k t} \equiv C_{K}^{k t}+C_{L}^{k t}$. Profit $\Pi^{k t}$ is the balancing item and thus may be positive, negative, or zero. We are operating here outside the neoclassical framework where profit always equals zero due to the structural and behavioural assumptions involved.

\footnotetext{
$\overline{2}$ This section has been adapted from corresponding sections of Balk (2015), (2016).

3 "Consolidated" means that intra-unit deliveries are netted out. At the industry level, in some parts of the literature this is called "sectoral". At the economy level, "sectoral" output reduces to GDP plus imports, and "sectoral" intermediate input to imports. In terms of variables to be defined below, consolidation means that $C_{E M S}^{k k t}=R^{k k t}=0$.
} 
The KL-VA accounting identity then reads

$$
C_{K L}^{k t}+\Pi^{k t}=R^{k t}-C_{E M S}^{k t} \equiv V A^{k t}(k \in \mathcal{K}),
$$

where $V A^{k t}$ denotes value added, defined as revenue minus intermediate inputs cost. In this article it will always be assumed that $V A^{k t}>0{ }^{4}$

We now consider whether the ensemble of production units $\mathcal{K}$ can be considered as a consolidated production unit. Though aggregation basically is addition, adding-up the KLEMS-Y relations (1) over all the units would imply double-counting because of deliveries between units. To see this, it is useful to split intermediate input cost and revenue into two parts, respectively concerning units belonging to the ensemble $\mathcal{K}$ and units belonging to the rest of the world. Thus,

$$
C_{E M S}^{k t}=\sum_{k^{\prime} \in \mathcal{K}} C_{E M S}^{k^{\prime} k t}+C_{E M S}^{e k t}
$$

where $C_{E M S}^{k^{\prime} k t}$ is the cost of the intermediate inputs purchased by unit $k$ from unit $k^{\prime}$, and $C_{E M S}^{e k t}$ is the cost of the intermediate inputs purchased by unit $k$ from the world beyond the ensemble $\mathcal{K}$. Similarly,

$$
R^{k t}=\sum_{k^{\prime} \in \mathcal{K}} R^{k k^{\prime} t}+R^{k e t},
$$

where $R^{k k^{\prime} t}$ is the revenue obtained by unit $k$ from delivering to unit $k^{\prime}$, and $R^{k e t}$ is the revenue obtained by unit $k$ from delivering to units outside of $\mathcal{K}$. Adding up the KLEMS-Y relations (1) then delivers

$$
\begin{aligned}
\sum_{k \in \mathcal{K}} C_{K L}^{k t}+ & \sum_{k \in \mathcal{K}} \sum_{k^{\prime} \in \mathcal{K}} C_{E M S}^{k^{\prime} k t}+\sum_{k \in \mathcal{K}} C_{E M S}^{e k t}+\sum_{k \in \mathcal{K}} \Pi^{k t} \\
& =\sum_{k \in \mathcal{K}} \sum_{k^{\prime} \in \mathcal{K}} R^{k k^{\prime} t}+\sum_{k \in \mathcal{K}} R^{k e t} .
\end{aligned}
$$

If for all the tradeable commodities output prices are identical to input prices (which is ensured by National Accounting conventions), or if there are no deliveries between the production units (e.g., if $\mathcal{K}$ is a narrowly defined industry), then the two intra- $\mathcal{K}$-trade terms cancel, and the foregoing expression reduces to ${ }^{5}$

$\sum_{k \in \mathcal{K}} C_{K L}^{k t}+\sum_{k \in \mathcal{K}} C_{E M S}^{e k t}+\sum_{k \in \mathcal{K}} \Pi^{k t}=\sum_{k \in \mathcal{K}} R^{k e t}$.

\footnotetext{
${ }^{4}$ This is a necessary but innocuous assumption. Only in exceptional cases value added is non-positive, for instance when the accounting period is so short that revenue and intermediate inputs cost are booked in different periods. Value added is an accounting concept, without normative connotations. After all, value added must be used to pay for capital and labour expenses.

5 See Balk (2015, footnote 2) for the treatment of net taxes on intermediates.
}

Recall that capital assets and hours worked are unique for each production unit, which implies that primary input cost may simply be added over the units, without any fear for double-counting. Thus expression (6) is the KLEMS-Y accounting relation for the ensemble $\mathcal{K}$, considered as a consolidated production unit. The corresponding KL-VA relation is then

$\sum_{k \in \mathcal{K}} C_{K L}^{k t}+\sum_{k \in \mathcal{K}} \Pi^{k t}=\sum_{k \in \mathcal{K}} R^{k e t}-\sum_{k \in \mathcal{K}} C_{E M S}^{e k t}$,

which can be written as ${ }^{6}$

$C_{K L}^{\mathcal{K} t}+\Pi^{\mathcal{K} t}=R^{\mathcal{K} t}-C_{E M S}^{\mathcal{K} t} \equiv V A^{\mathcal{K} t}$.

where $C_{K L}^{\mathcal{K} t} \equiv \sum_{k \in \mathcal{K}} C_{K L}^{k t}, \Pi^{\mathcal{K} t} \equiv \sum_{k \in \mathcal{K}} \Pi^{k t}, R^{\mathcal{K} t} \equiv \sum_{k \in \mathcal{K}} R^{k e t}$, and $C_{E M S}^{\mathcal{K} t} \equiv \sum_{k \in \mathcal{K}} C_{E M S}^{e k t}$. One verifies immediately that

$V A^{\mathcal{K} t}=\sum_{k \in \mathcal{K}} V A^{k t}$.

The structural similarity between expressions (2) and (8), together with the additive relations between all their elements, is the reason why the KL-VA production model is the natural starting point for studying the relation between individual and aggregate measures of productivity change.

\section{Prerequisites}

For any production unit, real value added of period $t, R V A^{k}$ $(t, b)$, is nominal value added, $V A^{k t}$, divided by a suitable price index $P_{V A}^{k}(t, b)$, for period $t$ relative to a certain reference period $b$. Rearranging this definition gives

$$
V A^{k t}=P_{V A}^{k}(t, b) R V A^{k}(t, b)(k \in \mathcal{K}) .
$$

Nominal value added is here as it were decomposed into a price component and a quantity component. Without loss of generality it may be assumed that period $b$ lies somewhere in the past and that the ensemble $\mathcal{K}$ already existed in period $b$. The functional form of the price indices may vary over the production units; in particular, the price indices may be direct or chained or mixed. It is assumed that $P_{V A}^{k}(b, b)=1$, so that $R V A^{k}(b, b)=V A^{k b}(k \in \mathcal{K})$; that is, at the reference period real value added is identical to nominal value added.

For the ensemble, considered as a higher-level production unit, we have a similar relation,

$$
V A^{\mathcal{K} t}=P_{V A}^{\mathcal{K}}(t, b) R V A^{\mathcal{K}}(t, b)
$$

\footnotetext{
${ }^{6}$ If $\mathcal{K}$ is an economy and $\Pi^{\mathcal{K} t}=0$ then this expression reduces to the familiar identity of gross domestic income and gross domestic product.
} 
where $P_{V A}^{\mathcal{K}}(t, b)$ is a value-added based price index for the ensemble $\mathcal{K}$ for period $t$ relative to the reference period $b$. For the time being it is sufficient to assume that this index is estimated from (a sample of) the data underlying the individual price indices $P_{V A}^{k}(t, b)(k \in \mathcal{K})$.

The additivity of nominal value added implies a restriction on the functional form of $P_{V A}^{\mathcal{K}}(t, b)$, which can be seen as follows. Substituting expressions (10) and (11) into the fundamental adding-up relation (9) and dividing both sides by real value added of the ensemble, $R V A^{\mathcal{K}}(t, b)$, delivers a relation between the price index for the ensemble and the individual price indices,

$P_{V A}^{\mathcal{K}}(t, b)=\sum_{k \in \mathcal{K}} \frac{R V A^{k}(t, b)}{R V A^{\mathcal{K}}(t, b)} P_{V A}^{k}(t, b)$.

It is also important to observe that, unlike nominal value added - see again expression (9) -, real value added generally appears to be not additive. The dual to expression (12) is

$$
R V A^{\mathcal{K}}(t, b)=\sum_{k \in \mathcal{K}} \frac{P_{V A}^{k}(t, b)}{P_{V A}^{\mathcal{K}}(t, b)} R V A^{k}(t, b) .
$$

For any individual production unit, the real primary input of period $t, X_{K L}^{k}(t, b)$, is defined as nominal primary input cost, $C_{K L}^{k t}$, divided by a suitable price index $P_{K L}^{k}(t, b)$ for period $t$ relative to the reference period $b$. Rearranging this definition gives

$C_{K L}^{k t}=P_{K L}^{k}(t, b) X_{K L}^{k}(t, b)(k \in \mathcal{K})$.

The corresponding relation for the ensemble reads

$C_{K L}^{\mathcal{K} t}=P_{K L}^{\mathcal{K}}(t, b) X_{K L}^{\mathcal{K}}(t, b)$,

where $C_{K L}^{\mathcal{K} t} \equiv \sum_{k \in \mathcal{K}^{t}} C_{K L}^{k t}$ and $P_{K L}^{\mathcal{K}}(t, b)$ is a suitable deflator for the primary input cost of the ensemble $\mathcal{K}$. The additivity of nominal primary input cost then implies that

$P_{K L}^{\mathcal{K}}(t, b)=\sum_{k \in \mathcal{K}} \frac{X_{K L}^{k}(t, b)}{X_{K L}^{\mathcal{K}}(t, b)} P_{K L}^{k}(t, b)$.

It is also important to observe that, unlike nominal primary input cost, real primary input generally appears to be not additive. The dual to expression (16) is

$X_{K L}^{\mathcal{K}}(t, b)=\sum_{k \in \mathcal{K}} \frac{P_{K L}^{k}(t, b)}{P_{K L}^{\mathcal{K}}(t, b)} X_{K L}^{k}(t, b)$.

\section{Decomposing value-added based total factor productivity change}

Value-added based total factor productivity (TFP) is defined as real value added divided by real primary input; that is, for the individual production units,

$\operatorname{TFPROD}_{V A}^{k}(t, b) \equiv \frac{R V A^{k}(t, b)}{X_{K L}^{k}(t, b)}(k \in \mathcal{K})$

and for the aggregate,

$\operatorname{TFPROD}_{V A}^{\mathcal{K}}(t, b) \equiv \frac{R V A^{\mathcal{K}}(t, b)}{X_{K L}^{\mathcal{K}}(t, b)}$.

An interesting interpretation of value-added based TFP is obtained by substituting expression (14) into expression (18). This yields

$\operatorname{TFPROD}_{V A}^{k}(t, b)=\frac{P_{K L}^{k}(t, b)}{C_{K L}^{k t} / R V A^{k}(t, b)}(k \in \mathcal{K}) ;$

that is, primary input price divided by unit cost, both normalized to reference period $b$ (see also Balk 2018b, 92). If profit equals zero then unit cost equals value-added based price index, and primal TFP equals dual TFP (defined as input price index divided by output price index).

Going from (an earlier) period $t^{\prime}$ to (a later) period $t$, individual TFP change is measured by the ratios $\operatorname{TFPROD}_{V A}^{k}(t, b) /$ TFPROD $_{V A}^{k}\left(t^{\prime}, b\right)(k \in \mathcal{K})$, and aggregate TFP change by TFPROD $D_{V A}^{\mathcal{K}}(t, b) / \operatorname{TFPROD}_{V A}^{\mathcal{K}}\left(t^{\prime}, b\right)$. Can the last ratio be written as a function of all the productionunit-specific ratios? ${ }^{7}$ Balk (2015, expressions (20), (28), and (34)) developed three (time-period-) symmetric decompositions of the aggregate TFP index. We will now show that there is a fourth decomposition.

To start with, the aggregate nominal value-added ratio, for period $t$ relative to period $t^{\prime}$, can be decomposed as

$\ln \left(\frac{V A^{\mathcal{K} t}}{V A^{\mathcal{K} t^{\prime}}}\right)=\sum_{k \in \mathcal{K}} \psi^{k}\left(t, t^{\prime}\right) \ln \left(\frac{V A^{k t}}{V A^{k t^{\prime}}}\right)$,

where

$\psi^{k}\left(t, t^{\prime}\right) \equiv \frac{L M\left(\frac{V A^{k t}}{V A^{K t}}, \frac{V A^{k t^{\prime}}}{V A^{k t^{\prime}}}\right)}{\sum_{k \in \mathcal{K}} L M\left(\frac{V A^{k t}}{V A^{K t}}, \frac{V A^{k t^{\prime}}}{V A^{K t^{\prime}}}\right)}(k \in \mathcal{K})$,

${ }^{7}$ Recall that the logarithm of any such ratio, if in the neighbourhood
of 1 , can be interpreted as a growth rate. 
and the function $\operatorname{LM(.)}$ is the logarithmic mean. ${ }^{8}$ Aggregate value-added change, measured as a ratio, is thus equal to a weighted geometric mean of individual value-added changes. Notice that the coefficients $\psi^{k}\left(t, t^{\prime}\right)$ add up to 1 . Each coefficient is the (normalized) mean share of production unit $k$ in aggregate nominal value added.

Similarly, the aggregate primary input cost ratio, for period $t$ relative to period $t^{\prime}$, can be decomposed as

$\ln \left(\frac{C_{K L}^{\mathcal{K} t}}{C_{K L}^{\mathcal{K} t^{\prime}}}\right)=\sum_{k \in \mathcal{K}} \omega^{k}\left(t, t^{\prime}\right) \ln \left(\frac{C_{K L}^{k t}}{C_{K L}^{k t^{\prime}}}\right)$

where

$$
\omega^{k}\left(t, t^{\prime}\right) \equiv \frac{L M\left(\frac{C_{K L}^{k t}}{C_{K L}^{\mathcal{K} t}}, \frac{C_{K L}^{k t^{\prime}}}{C_{K L}^{K t^{\prime}}}\right)}{\sum_{k \in \mathcal{K}} L M\left(\frac{C_{K L}^{k t}}{C_{K L}^{K L}}, \frac{C_{K L}^{k t^{\prime}}}{C_{K L}^{K t^{\prime}}}\right)}(k \in \mathcal{K}) .
$$

Aggregate primary-input cost change is thus equal to a weighted geometric mean of individual primary-input cost changes. Notice that the coefficients $\omega^{k}\left(t, t^{\prime}\right)$ add up to 1 . Each coefficient is the (normalized) mean share of production unit $k$ in aggregate primary-input cost.

Substituting the expressions (10) and (11) into (21), and substituting the expressions (14) and (15) into (22) delivers, respectively,

$\ln \left(\frac{P_{V A}^{\mathcal{K}}(t, b) R V A^{\mathcal{K}}(t, b)}{P_{V A}^{\mathcal{K}}\left(t^{\prime}, b\right) R V A^{\mathcal{K}}\left(t^{\prime}, b\right)}\right)=\sum_{k \in \mathcal{K}} \psi^{k}\left(t, t^{\prime}\right) \ln \left(\frac{P_{V A}^{k}(t, b) R V A^{k}(t, b)}{P_{V A}^{k}\left(t^{\prime}, b\right) R V A^{k}\left(t^{\prime}, b\right)}\right)$,

and

$$
\ln \left(\frac{P_{K L}^{\mathcal{K}}(t, b) X_{K L}^{\mathcal{K}}(t, b)}{P_{K L}^{\mathcal{K}}\left(t^{\prime}, b\right) X_{K L}^{\mathcal{K}}\left(t^{\prime}, b\right)}\right)=\sum_{k \in \mathcal{K}} \omega^{k}\left(t, t^{\prime}\right) \ln \left(\frac{P_{K L}^{k}(t, b) X_{K L}^{k}(t, b)}{P_{K L}^{k}\left(t^{\prime}, b\right) X_{K L}^{k}\left(t^{\prime}, b\right)}\right) .
$$

Subtracting Eq. (24) from Eq. (23), moving the aggregate price indices from the left-hand side to the right-hand side, using the fact that the coefficients add up to 1, and applying definition (19), delivers

$$
\begin{gathered}
\ln \left(\frac{\operatorname{TFPROD}_{V A}^{\mathcal{K}}(t, b)}{\operatorname{TFPROD}_{V A}^{K}\left(t^{\prime}, b\right)}\right)=\sum_{k \in \mathcal{K}} \psi^{k}\left(t, t^{\prime}\right) \ln \left(\frac{R V A^{k}(t, b)}{R V A^{k}\left(t^{\prime}, b\right)}\right)-\sum_{k \in \mathcal{K}} \omega^{k}\left(t, t^{\prime}\right) \ln \left(\frac{X_{K L}^{k}(t, b)}{X_{K L}^{k}\left(t^{\prime}, b\right)}\right)+ \\
\sum_{k \in \mathcal{K}} \psi^{k}\left(t, t^{\prime}\right) \ln \left(\frac{P_{V A}^{k}(t, b) / P_{V A}^{\mathcal{K}}(t, b)}{P_{V A}^{k}\left(t^{\prime}, b\right) / P_{V A}^{K}\left(t^{\prime}, b\right)}\right)-\sum_{k \in \mathcal{K}} \omega^{k}\left(t, t^{\prime}\right) \ln \left(\frac{P_{K L}^{k}(t, b) / P_{K L}^{\mathcal{K}}(t, b)}{P_{K L}^{k}\left(t^{\prime}, b\right) / P_{K L}^{\mathcal{K}}\left(t^{\prime}, b\right)}\right) .
\end{gathered}
$$

\footnotetext{
8 The logarithmic mean is, for any two strictly positive real numbers $a$ and $b$, defined by $L M(a, b) \equiv(a-b) / \ln (a / b)$ if $a \neq b$ and $L M(a, a) \equiv a$. It has the following properties: (1) $\min (a, b) \leq L M(a, b) \leq \max (a, b)$; (2) $L M(a, b)$ is continuous; (3) $L M(\lambda a, \lambda b)=\lambda L M(a, b)(\lambda>0)$; (4) $L M$ $(a, b)=L M(b, a) ;(5)(a b)^{1 / 2} \leq L M(a, b) \leq(a+b) / 2$; (6) $L M(a, 1)$ is concave. See Balk (2008) for details.
}

The last line of expression (25) concerns mean relative price change at the output side minus mean relative price change at the input side of the production units. Let this factor be denoted by $\ln P_{r e l}\left(t, t^{\prime}\right)$. If there is no relative price change at all, that is, $P_{V A}^{k}(t, b)=P_{V A}^{\mathcal{K}}(t, b)$ and $P_{K L}^{k}(t, b)=P_{K L}^{\mathcal{K}}(t, b)$ for all $k \in \mathcal{K}$ and all time periods considered, then $\ln P_{\text {rel }}\left(t, t^{\prime}\right)$ $=0$. However, such a situation is unlikely to occur.

The following observation is more interesting. If

$\ln \left(\frac{P_{V A}^{\mathcal{K}}(t, b)}{P_{V A}^{\mathcal{K}}\left(t^{\prime}, b\right)}\right)=\sum_{k \in \mathcal{K}} \psi^{k}\left(t, t^{\prime}\right) \ln \left(\frac{P_{V A}^{k}(t, b)}{P_{V A}^{k}\left(t^{\prime}, b\right)}\right)$

and

$\ln \left(\frac{P_{K L}^{\mathcal{K}}(t, b)}{P_{K L}^{\mathcal{K}}\left(t^{\prime}, b\right)}\right)=\sum_{k \in \mathcal{K}} \omega^{k}\left(t, t^{\prime}\right) \ln \left(\frac{P_{K L}^{k}(t, b)}{P_{K L}^{k}\left(t^{\prime}, b\right)}\right)$

then $\ln P_{r e l}\left(t, t^{\prime}\right)=0$. Technically, the assumptions expressed in the foregoing two expressions mean that the price indices for aggregate value added and primary input are (second-stage) Sato-Vartia $(\mathrm{S}-\mathrm{V})$ indices of the price indices for the individual production units. On the properties of the S-V indices, see Balk (2008). As such, these two expressions provide specifications of expressions (12) and (16), respectively.

The second line of expression (25) can be decomposed in several ways. Applying definition (18), the entire expression can be written either as

$$
\begin{gathered}
\ln \left(\frac{\operatorname{TFPROD}_{V A}^{\mathcal{K}}(t, b)}{\operatorname{TFPROD}_{V A}^{K}\left(t^{\prime}, b\right)}\right)=\sum_{k \in \mathcal{K}} \psi^{k}\left(t, t^{\prime}\right) \ln \left(\frac{\operatorname{TFPROD}_{V A}^{k}(t, b)}{\operatorname{TFPROD}_{V A}^{k}\left(t^{\prime}, b\right)}\right)+ \\
\sum_{k \in \mathcal{K}}\left(\psi^{k}\left(t, t^{\prime}\right)-\omega^{k}\left(t, t^{\prime}\right)\right)\left(\ln \left(\frac{X_{K L}^{k}(t, b)}{X_{K L}^{k}\left(t^{\prime}, b\right)}\right)-a^{\prime}\right)+\ln P_{r e l}\left(t, t^{\prime}\right),
\end{gathered}
$$

or as

$$
\begin{gathered}
\ln \left(\frac{\operatorname{TFPROD}_{V A}^{K}(t, b)}{\operatorname{TFPROD}_{V A}^{K}\left(t^{\prime}, b\right)}\right)=\sum_{k \in \mathcal{K}} \omega^{k}\left(t, t^{\prime}\right) \ln \left(\frac{\operatorname{TFPROD}_{V A}^{k}(t, b)}{\operatorname{TFPROD}_{V A}^{k}\left(t^{\prime}, b\right)}\right)+ \\
\sum_{k \in \mathcal{K}}\left(\psi^{k}\left(t, t^{\prime}\right)-\omega^{k}\left(t, t^{\prime}\right)\right)\left(\ln \left(\frac{R V A^{k}(t, b)}{R V A^{k}\left(t^{\prime}, b\right)}\right)-a^{\prime \prime}\right)+\ln P_{r e l}\left(t, t^{\prime}\right),
\end{gathered}
$$

or as the arithmetic mean of the former two expressions,

$$
\begin{gathered}
\ln \left(\frac{\operatorname{TFPROD}_{V A}^{\mathcal{K}}(t, b)}{\operatorname{TFPROD}_{V A}^{K}\left(t^{\prime}, b\right)}\right)=\sum_{k \in \mathcal{K}} \frac{1}{2}\left(\psi^{k}\left(t, t^{\prime}\right)+\omega^{k}\left(t, t^{\prime}\right)\right) \ln \left(\frac{\operatorname{TFPROD}_{V A}^{k}(t, b)}{\operatorname{TFPROD}_{V A}^{k}\left(t^{\prime}, b\right)}\right) \\
+\sum_{k \in \mathcal{K}}\left(\psi^{k}\left(t, t^{\prime}\right)-\omega^{k}\left(t, t^{\prime}\right)\right)\left(\ln \left(\frac{R V A^{k}(t, b)}{R V A^{k}\left(t^{\prime}, b\right)} \frac{X_{K L}^{k}(t, b)}{X_{K L}^{k L}\left(t^{\prime}, b\right)}\right)^{1 / 2}-a^{\prime \prime \prime}\right) \\
+\ln P_{r e l}\left(t, t^{\prime}\right),
\end{gathered}
$$


where $a^{\prime}, a^{\prime \prime}$ and $a^{\prime \prime \prime}$ are arbitrary scalars. Either of the expressions (28)-(30) constitutes the fourth decomposition. In each case aggregate TFP change consists of three main factors. The first is a (with respect to time) symmetrically weighted mean of the production-unit-specific TFP changes, where the weights in expression (28) are nominal-value-added shares, in expression (29) nominalprimary-input-cost shares, and in expression (30) the means of those shares. The second measures reallocation"; in expression (28) from the viewpoint of primary inputs, in expression (29) from the viewpoint of output (real value added), and in expression (30) from a combined viewpoint. The third, which is the same in the three expressions, measures net mean relative price change ${ }^{10}$, and vanishes if there is no relative price change or if $\mathrm{S}-\mathrm{V}$ indices are used, as in expressions (26) and (27).

Let us, by way of example, have a closer look at the reallocation factor in expression (28), and let this factor be denoted by $\ln R A L_{K L}\left(t, t^{\prime}\right)$. That indeed reallocation is being measured can be seen by selecting the arbitrary scalar as $a^{\prime}=\ln \left(X_{K L}^{\mathcal{K}}(t, b) / X_{K L}^{\mathcal{K}}\left(t^{\prime}, b\right)\right)$. Then the reallocation factor reduces to

$\ln R A L_{K L}\left(t, t^{\prime}\right)=\sum_{k \in \mathcal{K}}\left(\psi^{k}\left(t, t^{\prime}\right)-\omega^{k}\left(t, t^{\prime}\right)\right) \ln \left(\frac{X_{K L}^{k}(t, b) / X_{K L}^{\mathcal{K}}(t, b)}{X_{K L}^{k}\left(t^{\prime}, b\right) / X_{K L}^{\mathcal{K}}\left(t^{\prime}, b\right)}\right)$,

which measures the impact of the change of relative real primary input between the periods $t^{\prime}$ and $t$. Notice that the weights add up to 0 ; that is, $\sum_{k \in \mathcal{K}}\left(\psi^{k}\left(t, t^{\prime}\right)-\omega^{k}\left(t, t^{\prime}\right)\right)=0$. Thus the right-hand side of expression (31) is a covariance. A positive value of the reallocation factor means that primary inputs have moved to production units whose value-added share $\psi^{k}\left(t, t^{\prime}\right)$ is greater than their primary-input cost share $\omega^{k}\left(t, t^{\prime}\right){ }^{11}$

As real primary input is not additive, the relatives $X_{K L}^{k}(t, b) / X_{K L}^{\mathcal{K}}(t, b)(k \in \mathcal{K})$ do not add up to 1 . Shares can be obtained by selecting the arbitrary scalar as $a^{\prime}=\ln \left(\sum_{k \in \mathcal{K}} X_{K L}^{k}(t, b) / \sum_{k \in \mathcal{K}} X_{K L}^{k}\left(t^{\prime}, b\right)\right)$. Then the

\footnotetext{
9 There is a large literature on the topic of reallocation, but no universal definition of the concept. Though the word 'reallocation' seems to have a normative undertone, in the present context it can best be read as 'dynamics': the process of (relative) growth and decline of production units.

10 The occurrence of such a factor in a decomposition of aggregate productivity change was discussed in Balk (2015, Section 7). The central argument is that “... even if at the level of individual commodities the price is the same for every buyer/seller then the 'price' of the composite input and output commodity will vary over the production units."

${ }^{11}$ An alternative interpretation in terms of primary inputs moving to production units whose output per unit of primary inputs, $V A^{k t} / X_{K L}^{k}(t, b)$, is higher than average, $V A^{\mathcal{K} t} / X_{K L}^{\mathcal{K}}(t, b)$, as suggested by Bollard et al. (2013), holds only if $P_{K L}^{k}(t, b)=P_{K L}^{\mathcal{K}}(t, b)(k \in \mathcal{K})$.
}

reallocation factor reduces to

$\ln R A L_{K L}\left(t, t^{\prime}\right)=\sum_{k \in \mathcal{K}}\left(\psi^{k}\left(t, t^{\prime}\right)-\omega^{k}\left(t, t^{\prime}\right)\right) \ln \left(\frac{X_{K L}^{k}(t, b) / \sum_{k \in \mathcal{K}} X_{K L}^{k}(t, b)}{X_{K L}^{k}\left(t^{\prime}, b\right) / \sum_{k \in \mathcal{K}} X_{K L}^{k}\left(t^{\prime}, b\right)}\right)$.

By selecting the arbitrary scalar as $a^{\prime}=\sum_{k \in \mathcal{K}} \omega^{k}\left(t, t^{\prime}\right)$ $\ln \left(X_{K L}^{k}(t, b) / X_{K L}^{k}\left(t^{\prime}, b\right)\right)$ the reallocation factor appears to reduce to

$\ln R A L_{K L}\left(t, t^{\prime}\right)=\sum_{k \in \mathcal{K}} \psi^{k}\left(t, t^{\prime}\right) \ln \left(\frac{X_{K L}^{k}(t, b) / \prod_{k \in \mathcal{K}}\left(X_{K L}^{k}(t, b)\right)^{\omega^{k}\left(t, t^{\prime}\right)}}{X_{K L}^{k}\left(t^{\prime}, b\right) / \prod_{k \in \mathcal{K}}\left(X_{K L}^{k}\left(t^{\prime}, b\right)\right)^{\omega^{k}\left(t, t^{\prime}\right)}}\right)$.

Technically, $\exp \left\{a^{\prime}\right\}$ is now the Sato-Vartia quantity index of the individual primary input quantity indices $X_{K L}^{k}(t, b) / X_{K L}^{k}\left(t^{\prime}, b\right)(k \in \mathcal{K})$.

\section{Decomposing the reallocation factor into contributions of separate primary inputs}

The reallocation factor $\ln R A L_{K L}\left(t, t^{\prime}\right)$, as defined in the previous section, reads in terms of joint primary inputs capital $(K)$ and labour $(L)$. To see the contributions of these two input classes separately one needs some additional prerequisites.

The first is that there are separate, production-unit-specific deflators for nominal capital input cost and nominal labour input cost; that is, we have, analogous to expression (14),

$$
C_{K}^{k t}=P_{K}^{k}(t, b) X_{K}^{k}(t, b)(k \in \mathcal{K})
$$

and

$$
C_{L}^{k t}=P_{L}^{k}(t, b) X_{L}^{k}(t, b)(k \in \mathcal{K})
$$

where $P_{K}^{k}(t, b)$ and $P_{L}^{k}(t, b)$ are price indices and $X_{K}^{k}(t, b)$ and $X_{L}^{k}(t, b)$ are real inputs, for capital and labour respectively. As nominal primary input cost is additive $\left(C_{K L}^{k t}=C_{K}^{k t}+C_{L}^{k t}\right)$, it is clear that there must exist a relation between the joint price index $P_{K L}^{k}(t, b)$ and the separate price indices $P_{K}^{k}(t, b)$ and $P_{L}^{k}(t, b)$, or between joint real input $X_{K L}^{k}(t, b)$ and the separate real inputs $X_{K}^{k}(t, b)$ and $X_{L}^{k}(t, b)$.

The second assumption then concerns the way these relations are modeled. We here assume that joint real primary input is a convex combination of real capital and labour input; that is,

$$
X_{K L}^{k}(t, b) \equiv\left(X_{K}^{k}(t, b)\right)^{\alpha^{k}}\left(X_{L}^{k}(t, b)\right)^{1-\alpha^{k}}\left(0<\alpha^{k}<1 ; k \in \mathcal{K}\right)
$$


or

$$
\ln X_{K L}^{k}(t, b) \equiv \alpha^{k} \ln X_{K}^{k}(t, b)+\left(1-\alpha^{k}\right) \ln X_{L}^{k}(t, b)(k \in \mathcal{K}) .
$$

Then

$$
\begin{aligned}
\sum_{k \in \mathcal{K}} \omega^{k}\left(t, t^{\prime}\right) \ln X_{K L}^{k}(t, b) & =\sum_{k \in \mathcal{K}} \omega^{k}\left(t, t^{\prime}\right) \alpha^{k} \ln X_{K}^{k}(t, b)+\sum_{k \in \mathcal{K}} \omega^{k}\left(t, t^{\prime}\right)\left(1-\alpha^{k}\right) \ln X_{L}^{k}(t, b) \\
& =\alpha^{\mathcal{K}} \ln X_{K}^{\mathcal{K}}(t, b)+\left(1-\alpha^{\mathcal{K}}\right) \ln X_{L}^{\mathcal{K}}(t, b),
\end{aligned}
$$

where

$$
\begin{aligned}
& \alpha^{\mathcal{K}} \equiv \sum_{k \in \mathcal{K}} \omega^{k}\left(t, t^{\prime}\right) \alpha^{k} \\
& \ln X_{K}^{\mathcal{K}}(t, b) \equiv \sum_{k \in \mathcal{K}} \omega^{k}\left(t, t^{\prime}\right) \alpha^{k} \ln X_{K}^{k}(t, b) / \alpha^{\mathcal{K}} \\
& \ln X_{L}^{\mathcal{K}}(t, b) \equiv \sum_{k \in \mathcal{K}} \omega^{k}\left(t, t^{\prime}\right)\left(1-\alpha^{k}\right) \ln X_{L}^{k}(t, b) /\left(1-\alpha^{\mathcal{K}}\right) .
\end{aligned}
$$

The reallocation factor, as represented by expression (33), can then be written as

$$
\begin{gathered}
\ln R A L_{K L}\left(t, t^{\prime}\right)=\sum_{k \in \mathcal{K}} \psi^{k}\left(t, t^{\prime}\right)\left[\alpha^{k} \ln \left(\frac{X_{K}^{k}(t, b)}{X_{K}^{k}\left(t^{\prime}, b\right)}\right)-\alpha^{\mathcal{K}} \ln \left(\frac{X_{K}^{\mathcal{K}}(t, b)}{X_{K}^{\kappa}\left(t^{\prime}, b\right)}\right)\right] \\
+\sum_{k \in \mathcal{K}} \psi^{k}\left(t, t^{\prime}\right)\left[\left(1-\alpha^{k}\right) \ln \left(\frac{X_{L}^{k}(t, b)}{X_{L}^{k}\left(t^{\prime}, b\right)}\right)-\left(1-\alpha^{\mathcal{K}}\right) \ln \left(\frac{X_{L}^{\mathcal{K}}(t, b)}{X_{L}^{\mathcal{K}}\left(t^{\prime}, b\right)}\right)\right]
\end{gathered}
$$

where the contributions of the two primary input classes are nicely separated. Expression (42) bears a stark resemblance to the reallocation term figuring in the decomposition obtained by Baldwin et al. (2013, expression (10)).

Notice that expression (36) represents a production-unitspecific Cobb-Douglas aggregator function. This choice is not completely arbitrary, but its defense would require a separate paper. In conventional empirical work the $\alpha^{k}$ s are estimated and not production-unit-specific.

\section{Introducing gross-output based total factor productivity change}

At the right-hand side of expressions (28), (29) and (30) we see weighted means of production-unit-specific value-added based TFP change. As gross-output (or revenue) stays closer to the actual operations of a production unit, we want to replace value-added by gross-output based TFP change.

Gross-output based TFP is defined as real revenue divided by real KLEMS input; that is,

$$
\operatorname{TFPROD}_{Y}^{k}(t, b) \equiv \frac{Y^{k}(t, b)}{X_{K L E M S}^{k}(t, b)}(k \in \mathcal{K}),
$$

where nominal revenue is supposed to be decomposable as

$$
R^{k t}=P_{R}^{k}(t, b) Y^{k}(t, b)(k \in \mathcal{K})
$$

and nominal (total) cost as

$$
C^{k t} \equiv C_{K L}^{k t}+C_{E M S}^{k t}=P_{K L E M S}^{k}(t, b) X_{K L E M S}^{k}(t, b)(k \in \mathcal{K}) .
$$

Also nominal intermediate input cost is supposed to be decomposable as

$$
C_{E M S}^{k t}=P_{E M S}^{k}(t, b) X_{E M S}^{k}(t, b)(k \in \mathcal{K}) .
$$

In the above $P_{R}^{k}(t, b), P_{K L E M S}^{k}(t, b)$, and $P_{E M S}^{k}(t, b)$ are suitable deflators for nominal revenue, nominal (total) cost, and nominal intermediate input cost, respectively; and $Y^{k}(t$, $b), X_{K L E M S}^{k}(t, b)$, and $X_{E M S}^{k}(t, b)$ their real counterparts. Decompositions of primary input cost, $C_{K L}^{k t}$, and nominal value added, $V A^{k t}$, were already provided by expressions (14) and (10), respectively.

Based on the fact that nominal value added plus intermediate inputs cost equals revenue, $R^{k t}=V A^{k t}+C_{E M S}^{k t}$ $(k \in \mathcal{K})$, it is assumed that

$$
\begin{gathered}
\ln \left(\frac{Y^{k}(t, b)}{Y^{k}\left(t^{\prime}, b\right)}\right)=\frac{L M\left(V A^{k t}, V A^{k t^{\prime}}\right)}{L M\left(R^{k t}, R^{k t^{\prime}}\right)} \ln \left(\frac{R V A^{k}(t, b)}{R V A^{k}\left(t^{\prime}, b\right)}\right) \\
+\frac{L M\left(C_{E M S}^{k t}, C_{E M S}^{k t^{\prime}}\right)}{L M\left(R^{k t}, R^{k t^{\prime}}\right)} \ln \left(\frac{X_{E M S}^{k}(t, b)}{X_{E M S}^{k}\left(t^{\prime}, b\right)}\right)
\end{gathered}
$$

where $L M($.$) is the logarithmic mean. Basically this means$ that the revenue-based output quantity index for period $t$ relative to period $t^{\prime}$ is defined as the Montgomery-Vartia (M-V) index of the value-added based output quantity index and the intermediate inputs quantity index. On the properties of the M-V index, see Balk (2008). In particular one should notice that the weights do not add up to 1, due to the concavity of the logarithmic mean. Expression (47) is equivalent to the dual relation between the corresponding price indices,

$$
\begin{gathered}
\ln \left(\frac{P_{R}^{k}(t, b)}{P_{R}^{k}\left(t^{\prime}, b\right)}\right)=\frac{L K\left(V A^{k t}, V A^{k t^{\prime}}\right)}{L M\left(R^{k t}, R^{k t^{\prime}}\right)} \ln \left(\frac{P_{V A}^{k}(t, b)}{P_{V A}^{k}\left(t^{\prime}, b\right)}\right) \\
+\frac{L M\left(C_{E M S}^{k t}, C_{E M S}^{k t^{\prime}}\right)}{L M\left(R^{k t}, R^{k t^{\prime}}\right)} \ln \left(\frac{P_{E M S}^{k}(t, b)}{P_{E M S}^{k}\left(t^{\prime}, b\right)}\right)
\end{gathered}
$$

Expression (47) can be rearranged as

$$
\begin{gathered}
\ln \left(\frac{R V A^{k}(t, b)}{R V A^{k}\left(t^{\prime}, b\right)}\right)=\frac{L M\left(R^{k t}, R^{k t^{\prime}}\right)}{L M\left(V A^{k t}, V A^{k t^{\prime}}\right)} \ln \left(\frac{Y^{k}(t, b)}{Y^{k}\left(t^{\prime}, b\right)}\right) \\
-\frac{L M\left(C_{E M S}^{k t}, C_{E M S}^{k t^{\prime}}\right)}{L M\left(V A^{k t}, V A^{k t^{\prime}}\right)} \ln \left(\frac{X_{E M S}^{k}(t, b)}{X_{E M S}^{k}\left(t^{\prime}, b\right)}\right) .
\end{gathered}
$$

By substituting expression (49) into the ratio of value-added based TFP for period $t$ and period $t^{\prime}$, as defined by 
expression (18), we obtain

$$
\begin{aligned}
& \ln \left(\frac{\operatorname{TFPROD}_{V A}^{k}(t, b)}{\operatorname{TFPROD}_{V A}^{k}\left(t^{\prime}, b\right)}\right)=\frac{L M\left(R^{k t}, R^{k t^{\prime}}\right)}{L M\left(V A^{k t}, V A^{k t^{\prime}}\right)} \ln \left(\frac{Y^{k}(t, b)}{Y^{k}\left(t^{\prime}, b\right)}\right) \\
& -\frac{L M\left(C_{E M S}^{k t}, C_{E M S}^{k t^{\prime}}\right)}{L M\left(V A^{k t}, V A^{k t^{\prime}}\right)} \ln \left(\frac{X_{E M S}^{k}(t, b)}{X_{E M S}^{k}\left(t^{\prime}, b\right)}\right)-\ln \left(\frac{X_{K L}^{k}(t, b)}{X_{K L}^{k}\left(t^{\prime}, b\right)}\right) .
\end{aligned}
$$

Next, it is assumed that

$$
\begin{gathered}
\ln \left(\frac{X_{K L E M S}^{k}(t, b)}{X_{K L E M S}^{k}\left(t^{\prime}, b\right)}\right)=\frac{L M\left(C_{K L}^{k t}, C_{K L}^{k t^{\prime}}\right)}{L M\left(C^{k t}, C^{k t^{\prime}}\right)} \ln \left(\frac{X_{K L}^{k}(t, b)}{X_{K L}^{k}\left(t^{\prime}, b\right)}\right) \\
+\frac{L M\left(C_{E M S}^{k t}, C_{E M S}^{k t^{\prime}}\right)}{L M\left(C^{k t}, C^{k t^{\prime}}\right)} \ln \left(\frac{X_{E M S}^{k}(t, b)}{X_{E M S}^{k}\left(t^{\prime}, b\right)}\right)
\end{gathered}
$$

which means that the KLEMS input quantity index for period $t$ relative to period $t^{\prime}$ is defined as the $\mathrm{M}-\mathrm{V}$ index of the primary input quantity index and the intermediate inputs quantity index. Notice that expression (51) is equivalent to the dual relation between the corresponding price indices,

$$
\begin{gathered}
\ln \left(\frac{P_{K L E M S}^{k}(t, b)}{P_{K L E M S}^{k}\left(t^{\prime}, b\right)}\right)=\frac{L M\left(C_{K L}^{k t}, C_{K L}^{k t^{\prime}}\right)}{L M\left(C^{k t}, C^{k t^{\prime}}\right)} \ln \left(\frac{P_{K L}^{k}(t, b)}{P_{K L}^{k}\left(t^{\prime}, b\right)}\right) \\
+\frac{L M\left(C_{E M S}^{k t}, C_{E M S}^{k t^{\prime}}\right)}{L M\left(C^{k t}, C^{k t^{\prime}}\right)} \ln \left(\frac{P_{E M S}^{k}(t, b)}{P_{E M S}^{k}\left(t^{\prime}, b\right)}\right)
\end{gathered}
$$

By substituting expression (51) into the ratio of grossoutput based TFP for period $t$ and period $t^{\prime}$, as defined by expression (43), we obtain

$$
\begin{gathered}
\ln \left(\frac{\operatorname{TFPROD}_{Y}^{k}(t, b)}{\operatorname{TFPROD}_{Y}^{k}\left(t^{\prime}, b\right)}\right)=\ln \left(\frac{Y^{k}(t, b)}{Y^{k}\left(t^{\prime}, b\right)}\right)-\frac{L M\left(C_{K L}^{k t}, C_{K L}^{k t^{\prime}}\right)}{L M\left(C^{k t}, C^{k t^{\prime}}\right)} \ln \left(\frac{X_{K L}^{k}(t, b)}{X_{K L}^{k}\left(t^{\prime}, b\right)}\right) \\
-\frac{L M\left(C_{E M S}^{k t}, C_{E M S}^{k t^{\prime}}\right)}{L M\left(C^{k t}, C^{k t^{\prime}}\right)} \ln \left(\frac{X_{E M S}^{k}(t, b)}{X_{E M S}^{k}\left(t^{\prime}, b\right)}\right),
\end{gathered}
$$

or

$$
\begin{aligned}
\ln \left(\frac{Y^{k}(t, b)}{Y^{k}\left(t^{\prime}, b\right)}\right)= & \ln \left(\frac{\operatorname{TFPROD}_{Y}^{k}(t, b)}{\operatorname{TFPROD}_{Y}^{k}\left(t^{\prime}, b\right)}\right)+\frac{L M\left(C_{K L}^{k t}, C_{K L}^{k t^{\prime}}\right)}{L M\left(C^{k t}, C^{k t^{\prime}}\right)} \ln \left(\frac{X_{K L}^{k}(t, b)}{X_{K L}^{k}\left(t^{\prime}, b\right)}\right) \\
& +\frac{L M\left(C_{E M S}^{k t}, C_{E M S}^{k t^{\prime}}\right)}{L M\left(C^{k t}, C^{k t^{\prime}}\right)} \ln \left(\frac{X_{E M S}^{k}(t, b)}{X_{E M S}^{k}\left(t^{\prime}, b\right)}\right) .
\end{aligned}
$$

Substituting expression (54) into expression (50) finally delivers

$$
\begin{gathered}
\ln \left(\frac{T_{\text {TFPROD }}^{k}(t, b)}{\operatorname{TFPROD}_{V A}^{k}\left(t^{\prime}, b\right)}\right)=\frac{L M\left(R^{k t}, R^{k t^{\prime}}\right)}{L M\left(V A^{k t}, V A^{k t^{\prime}}\right)}\left[\ln \left(\frac{\operatorname{TFPROD}_{Y}^{k}(t, b)}{\operatorname{TFPROD}_{Y}^{k}\left(t^{\prime}, b\right)}\right)\right. \\
+\left(\frac{L M\left(C_{K L}^{k t}, C_{K L}^{k t^{\prime}}\right)}{L M\left(C^{k t}, C^{k t^{\prime}}\right)}-\frac{L M\left(V A^{k t}, V A^{k t^{\prime}}\right)}{L M\left(R^{k t}, R^{k t^{\prime}}\right)}\right) \ln \left(\frac{X_{K L}^{k}(t, b)}{X_{K L}^{k}\left(t^{\prime}, b\right)}\right) \\
\left.+\left(\frac{L M\left(C_{E M S}^{k t}, C_{E M S}^{k t^{\prime}}\right)}{L M\left(C^{k t}, C^{k t^{\prime}}\right)}-\frac{L M\left(C_{E M S}^{k t}, C_{E M S}^{k t^{\prime}}\right)}{L M\left(R^{k t}, R^{k t^{\prime}}\right)}\right) \ln \left(\frac{X_{E M S}^{k}(t, b)}{X_{E M S}^{k}\left(t^{\prime}, b\right)}\right)\right],
\end{gathered}
$$

which corresponds with the formula obtained by Balk (2009) for the first time. The factor in front of the square brackets, $L M\left(R^{k t}, R^{k t^{\prime}}\right) / L M\left(V A^{k t}, V A^{k t^{\prime}}\right)$, is known as the Domar factor: the ratio of (mean) nominal revenue over (mean) nominal value added.

An alternative decomposition of value-added based TFP change in terms of gross-output based TFP change plus some additional factors was obtained by Basu and Fernald (2002). It is possible to mimick their derivation in our setup; however, their avoidance of the Domar factor leads to a final expression which, though containing the same factors as our expression (55) - real primary input change and real intermediate input change - exhibits more complicated weights.

It is useful to recall the specific assumptions made in the course of the derivation of expression (55):

- For each production unit, the revenue-based output quantity index is an M-V index of the value-added based output quantity index and the primary input quantity index.

- For each production unit, the total input quantity index is an $\mathrm{M}-\mathrm{V}$ index of the primary input quantity index and the intermediate inputs quantity index.

The functional forms of the quantity indices for value added, primary input, and intermediate inputs are left unspecified. However, if these indices were themselves M-V indices of the underlying price and quantity data then, due to the consistency-in-aggregation of $\mathrm{M}-\mathrm{V}$ indices, both the revenue-based output quantity index and the total input quantity index would be $\mathrm{M}-\mathrm{V}$ indices of the underlying data.

Further, as Diewert (1978) has shown, at any given data point an $\mathrm{M}-\mathrm{V}$ index differentially approximates to the second order any other time-symmetric index, such as Fisher or Törnqvist. Thus, if for revenue-based output quantity and total input quantity instead of $\mathrm{M}-\mathrm{V}$ indices other timesymmetric indices were used, then the equality sign in expression (55) must be replaced by an approximation sign. In the limit, that is, if period $t^{\prime}$ approaches period $t$, then appproximation tends to equality. ${ }^{12}$

\section{The zero profit case}

It is important to consider what happens if for all the production units at any time period profit equals zero; that is, $\Pi^{k t}=0(k \in \mathcal{K})$. Such a situation materializes if the unit

\footnotetext{
${ }^{12}$ Diewert (2015) replaced the $\mathrm{M}-\mathrm{V}$ indices in the two expressions (47) and (51) by Laspeyres and Paasche indices, which are only firstorder differential approximations, and found that, under the zero-profit condition discussed below, the ratio of value-added based and grossoutput based TFP growth rates approximates the asymmetric Domar factors, $R^{k t^{\prime}} / V A^{k t^{\prime}}$ and $R^{k t} / V A^{k t}$, respectively. Two further assumptions, namely that geometric means can be approximated by arithmetic means and that Laspeyres and Paasche revenue-based output quantity indices are equal, made it possible to obtain a similar result in the case of Fisher indices. It is left to the reader to judge whether Diewert's derivation method is "much simpler" than mine. Using Australian data, Calver (2015) presents evidence on the variability of the Domar factors over industries and through time and on the accuracy of the approximations.
} 
user cost of all the capital assets is based on endogenous interest rates (which, then, are production-unit-specific), or if actual profit is considered as cost of an additional input called enterpreneurial activity (the price of which, then, is production-unit-specific). Zero profit is easily seen to be equivalent to $R^{k t}=C^{k t}$ or $V A^{k t}=C_{K L}^{k t}(k \in \mathcal{K})$.

The first consequence is that the coefficients $\psi^{k}\left(t, t^{\prime}\right)$ and $\omega^{k}\left(t, t^{\prime}\right)(k \in \mathcal{K})$ are identical, so that expressions (28), (29) and (30) reduce to

$$
\begin{aligned}
\ln \left(\frac{\operatorname{TFPROD}_{V A}^{K}(t, b)}{\operatorname{TFPROD}_{V A}\left(t^{\prime}, b\right)}\right) & =\sum_{k \in \mathcal{K}} \psi^{k}\left(t, t^{\prime}\right) \ln \left(\frac{\operatorname{TFPROD}_{V A}^{k}(t, b)}{\operatorname{TFPROD}_{V A}\left(t^{\prime}, b\right)}\right) \\
& +\ln P_{r e l}\left(t, t^{\prime}\right) .
\end{aligned}
$$

Quite surprisingly, we conclude that the entire reallocation factor has vanished.

The second consequence, easily checked, is that expression (55) reduces to

$\ln \left(\frac{\operatorname{TFPROD}_{V A}^{k}(t, b)}{\operatorname{TFPROD}_{V A}^{k}\left(t^{\prime}, b\right)}\right)=\frac{L M\left(R^{k t}, R^{k t^{\prime}}\right)}{L M\left(V A^{k t}, V A^{k t^{\prime}}\right)} \ln \left(\frac{\operatorname{TFPROD}_{Y}^{k}(t, b)}{\operatorname{TFPROD}_{Y}^{k}\left(t^{\prime}, b\right)}\right)(k \in \mathcal{K})$.

Notice that under the zero profit condition the Domar factors may alternatively be expressed as $L M\left(C^{k t}, C^{k t^{\prime}}\right) / L M\left(C_{K L}^{k t}, C_{K L}^{k t^{\prime}}\right)(k \in \mathcal{K})$; that is, reciprocals of (mean) primary input cost shares. Expression (57) means, put in words, that value-added based TFP growth equals gross-output based TFP growth times the Domar factor. ${ }^{13}$

By substituting expression (57) into expression (56), one obtains

$$
\begin{aligned}
\ln \left(\frac{\operatorname{TFPROD}_{V A}^{\mathcal{K}}(t, b)}{\operatorname{TFPROD}_{V A}\left(t^{\prime}, b\right)}\right) & =\sum_{k \in \mathcal{K}} D^{k}\left(t, t^{\prime}\right) \ln \left(\frac{\operatorname{TFPROD}_{Y}^{k}(t, b)}{\operatorname{TFPROD}_{Y}^{k}\left(t^{\prime}, b\right)}\right) \\
& +\ln P_{\text {rel }}\left(t, t^{\prime}\right),
\end{aligned}
$$

where the coefficients $D^{k}\left(t, t^{\prime}\right) \equiv \psi^{k}\left(t, t^{\prime}\right)\left(L M\left(R^{k t}, R^{k t^{\prime}}\right) / L M\right.$ $\left.\left(V A^{k t}, V A^{k t^{\prime}}\right)\right)(k \in \mathcal{K})$ measure (mean) individual nominal revenue over (mean) aggregate nominal value added; they are known as Domar weights. Their sum is greater than or equal to 1 . Following conventional wisdom, this reflects "the fact that an increase in the growth of the industry's productivity has two effects: the first is a direct effect on the industry's output and the second an indirect effect via the output delivered to other industries as intermediate inputs." (Jorgenson 2018, 881) Our derivation, however, makes clear that it is nothing but a mathematical artefact, caused by moving intermediate inputs cost from the denominator of a gross-output based productivity index to the numerator with a minus sign to get a value-added based productivity index.

\footnotetext{
13 A consequence is that the covariance of value-added based TFP growth and some other variable equals the covariance of gross-output based TFP growth and this variable times the Domar factor. It is good to keep this in mind when meeting such covariances in the literature on firm dynamics.
}

It is useful to summarize our findings in the form of a theorem.

Theorem 1 Let for any production unit $k \in \mathcal{K}$ suitable deflators for value added (VA), primary input $(K L)$, and intermediate inputs (EMS) be given: $P_{V A}^{k}(t, b), P_{K L}^{k}(t, b)$, and $P_{E M S}^{k}(t, b)$, respectively. Let the deflator for revenue, $P_{R}^{k}(t, b)$, be a $M-V$ index of $P_{V A}^{k}(t, b)$ and $P_{E M S}^{k}(t, b)$, and let the deflator for total input cost, $P_{K L E M S}^{k}(t, b)$, be a $M-V$ index of $P_{K L}^{k}(t, b)$ and $P_{E M S}^{k}(t, b)$. Let the deflator for aggregate value added, $P_{V A}^{\mathcal{K}}(t, b)$, and the deflator for aggregate primary input cost, $P_{K L}^{\mathcal{K}}(t, b)$, be $S$-V indices of the corresponding production-unit-specific deflators $P_{V A}^{k}(t, b)$ and $P_{K L}^{k}(t, b)(k \in \mathcal{K})$, respectively. If for any production unit profit equals zero, that is, $\Pi^{k t}=0(k \in \mathcal{K})$, then aggregate value-added based TFP change is a Domarweighted product of production-unit-specific gross-output based TFP changes,

$$
\frac{\operatorname{TFPROD}_{V A}^{\mathcal{K}}(t, b)}{\operatorname{TFPROD}_{V A}^{\mathcal{K}}\left(t^{\prime}, b\right)}=\prod_{k \in \mathcal{K}}\left(\frac{\operatorname{TFPROD}_{Y}^{k}(t, b)}{\operatorname{TFPROD}_{Y}^{k}\left(t^{\prime}, b\right)}\right)^{D^{k}\left(t, t^{\prime}\right)} .
$$

In official statistical practice the assumptions concerning the use of M-V and S-V indices are not fulfilled because simpler indices such as Laspeyres or Fisher are used as deflators. Then expression (59) holds only approximately. The better the indices actually used approximate $\mathrm{M}-\mathrm{V}$ and $\mathrm{S}-\mathrm{V}$ indices the better the final approximation will be. As the accuracy of any approximation hinges on the variance, over time and over production units, of the underlying price and quantity data, closeness of the time periods compared and similarity of the production units involved are crucial for obtaining a good approximation.

\section{Going beyond total factor productivity change}

Recall that production-unit specific gross-output based TFP was defined by expression (43). Using the assumption incorporated in expression (51) we obtained expression (53), here repeated as

$$
\begin{gathered}
\ln \left(\frac{\text { TFPROD }_{Y}^{k}(t, b)}{\operatorname{TFPROD}_{Y}^{k}\left(t^{\prime}, b\right)}\right)=\ln \left(\frac{Y^{k}(t, b)}{Y^{k}\left(t^{\prime}, b\right)}\right)-\vartheta_{K L}^{k t t^{\prime}} \ln \left(\frac{X_{K L}^{k}(t, b)}{X_{K L}^{k}\left(t^{\prime}, b\right)}\right) \\
-\vartheta_{E M S}^{k t t^{\prime}} \ln \left(\frac{X_{E M S}^{k}(t, b)}{X_{E M S}^{k}\left(t^{\prime}, b\right)}\right)(k \in \mathcal{K}),
\end{gathered}
$$

in which $\vartheta_{K L}^{k t t^{\prime}} \equiv L M\left(C_{K L}^{k t}, C_{K L}^{k t^{\prime}}\right) / L M\left(C^{k t}, C^{k t^{\prime}}\right)$ and $\vartheta_{E M S}^{k t t^{\prime}} \equiv$ $L M\left(C_{E M S}^{k t}, C_{E M S}^{k t^{\prime}}\right) / L M\left(C^{k t}, C^{k t^{\prime}}\right)(k \in \mathcal{K})$. Expression (60) is an example of the Solow residual: the growth rate of aggregate output minus a weighted mean of the growth rates of aggregate primary and intermediate inputs. However, as we did not introduce the usual neoclassical assumptions we 
cannot consider the Solow residual as a measure of technological change, or the impact of innovation (as Jorgenson 2018 does).

In the absence of such assumptions, the Solow residual is what it is. In order to make progress we need to decompose the residual into economically meaningful components representing technical efficiency change, technological change, scale effects, and input and output mix effects. For this we need to assume the existence of a time-period-specific technology to which the production units belonging to the ensemble $\mathcal{K}$ have access, with features so regular that analytical techniques can be used, and which can be estimated from available data. It is beyond the scope of this article to explore this topic further; the reader is referred to Balk and Zofío (2018).

It might, however, be useful to provide a simple illustration. It is assumed that the technology can be represented by a simple, time-invariant Cobb-Douglas function; that is, we assume that

$$
Y^{k}(\tau, b)=\Omega^{k}(\tau, b)\left(X_{K L}^{k}(\tau, b)\right)^{\alpha_{K L}}\left(X_{E M S}^{k}(\tau, b)\right)^{\alpha_{E M S}}\left(k \in \mathcal{K}, \tau=t^{\prime}, t\right),
$$

where $0<\Omega^{k}(\tau, b) \leq 1$ measures the technical efficiency of production unit $k \in \mathcal{K}$.

By substituting expression (61) into expression (60) we obtain

$$
\begin{gathered}
\ln \left(\frac{\text { TFPROD }_{Y}^{k}(t, b)}{\operatorname{TFPROD}_{Y}^{k}\left(t^{\prime}, b\right)}\right)=\ln \left(\frac{\Omega^{k}(t, b)}{\Omega^{k}\left(t^{\prime}, b\right)}\right)+\left(\alpha_{K L}-\vartheta_{K L}^{k t t^{\prime}}\right) \ln \left(\frac{X_{K L}^{k}(t, b)}{X_{K L}^{k}\left(t^{\prime}, b\right)}\right) \\
+\left(\alpha_{E M S}-\vartheta_{E M S}^{k t t^{\prime}}\right) \ln \left(\frac{X_{E M S}^{k}(t, b)}{X_{E M S}^{k}\left(t^{\prime}, b\right)}\right)(k \in \mathcal{K}) .
\end{gathered}
$$

One immediately recognizes here the familiar components of an empirical measure of TFP change: the first factor on the right-hand side of expression (62) measures technical efficiency change, whereas the second and third factor measure scale-and-input-mix effects. These two factors vanish if the empirical cost shares $\vartheta_{K L}^{k t t^{\prime}}$ and $\vartheta_{E M S}^{k t t^{\prime}}$-which, as we know, approximately add up to 1 -coincide with the elasticities $\alpha_{K L}$ and $\alpha_{E M S}$-which add up to 1 if constant returns to scale is assumed -, respectively. There is no role for technological change, as the production function is assumed to be time-invariant.

By substituting expression (62) into expression (58) we obtain for aggregate value-added based TFP change the following decomposition:

$$
\begin{aligned}
& \ln \left(\frac{\operatorname{TFPROD}_{V A}^{\mathcal{K}}(t, b)}{\operatorname{TFPROD}_{V A}^{\mathcal{K}}\left(t^{\prime}, b\right)}\right)=\sum_{k \in \mathcal{K}} D^{k}\left(t, t^{\prime}\right) \ln \left(\frac{\Omega^{k}(t, b)}{\Omega^{k}\left(t^{\prime}, b\right)}\right) \\
& +\sum_{k \in \mathcal{K}} D^{k}\left(t, t^{\prime}\right)\left(\alpha_{K L}-\vartheta_{K L}^{k t t^{\prime}}\right) \ln \left(\frac{X_{K L}^{k}(t, b)}{X_{K L}^{k}\left(t^{\prime}, b\right)}\right) \\
& +\sum_{k \in \mathcal{K}} D^{k}\left(t, t^{\prime}\right)\left(\alpha_{E M S}-\vartheta_{E M S}^{k t t^{\prime}}\right) \ln \left(\frac{X_{E M S}^{k}(t, b)}{X_{E M S}^{k}\left(t^{\prime}, b\right)}\right)+\ln P_{r e l}\left(t, t^{\prime}\right) .
\end{aligned}
$$

Apart from some details, such as the possible role of fixed costs and the relative price change factor, I believe this expression corresponds to the decomposition advocated by Petrin and Levinsohn (2012). Petrin and Levinsohn called the second and third factor on the right-hand side reallocation. However, as we have seen already, reallocation has vanished as a result of the zero profit assumption. Hence, as indicated, it is more appropriate to consider the second and third factor as measuring the aggregate effect of scale and input mix change. ${ }^{14}$

\section{Conclusion}

A key element in any system of productivity statistics comprising various levels of aggregation (economy, industry, firm) is a relation connecting a productivity index at a certain level to those at lower levels. In this article such a relation was derived, without invoking any of the usual neoclassical assumptions (a technology exhibiting constant returns to scale, competitive input and output markets, optimizing behaviour of the agents, and perfect foresight), just by mathematically manipulating the various accounting relations. In the process also the famous Domar factor could be demystified to being nothing but a mathematical artefact.

Our key relation links higher level value-added based productivity growth to a weighted sum of lower level productivity growth, a reallocation factor (reflecting the aggregate effect of lower level dynamics), and a relative price change factor. If zero profit is imposed, then the reallocation factor vanishes, and lower level value-added based productivity growth can be replaced by Domar weighted gross-output based productivity growth. Moreover, if the 'correct' deflators are used, then the relative price change factor also vanishes.

All this underscores the fact that by and large in empirical work, at various levels of aggregation, reallocation and relative price change tend to play a minor role visa-vis lower level productivity growth as such.

Acknowledgements The author thanks two referees whose comments, questions, and suggestions have led to several improvements.

\section{Compliance with ethical standards}

Conflict of interest The author declares that he has no conflict of interest.

\footnotetext{
${ }^{14}$ An important part of the Petrin and Levinsohn (2012) article was devoted to an empirical comparison of the decomposition in expression (63), minus the relative price change factor, with a concept called 'BHC productivity change'. However, the two concepts appear to measure different things, which makse a comparison rather meaningless.
} 
Publisher's note: Springer Nature remains neutral with regard to jurisdictional claims in published maps and institutional affiliations.

Open Access This article is distributed under the terms of the Creative Commons Attribution 4.0 International License (http://crea tivecommons.org/licenses/by/4.0/), which permits use, duplication, adaptation, distribution, and reproduction in any medium or format, as long as you give appropriate credit to the original author(s) and the source, provide a link to the Creative Commons license, and indicate if changes were made.

\section{References}

Baldwin JR, Gu W, Yan B (2013) Export growth, capacity utilization, and productivity growth: evidence from the Canadian manufacturing plants. Rev Income Wealth 59:665-688

Balk BM (2008) Price and Quantity Index Numbers: Models for Measuring Aggregate Change and Difference. Cambridge University Press, New York

Balk BM (2009) On the relation between gross-output and valueadded based productivity measures: The importance of the Domar factor Macroecon Dyn 13(Supplement 2):241-267

Balk BM (2010) An assumption-free framework for measuring productivity change Rev Income Wealth 56(Special Issue 1): S224-S256

Balk BM (2011) Measuring and decomposing capital input cost Rev Income Wealth 57:490-512

Balk BM (2014) Dissecting aggregate output and labour productivity change. J Prod Anal 42:35-43

Balk BM (2015) Measuring and relating aggregate and subaggregate total factor productivity change without neoclassical assumptions. Stat Neerl 69:21-48

Balk BM (2016) The Dynamics of Productivity Change: A Review of the Bottom-up Approach. In: Greene WH, Khalaf L, Sickles RC, Veall M, Voia M-C (eds) Productivity and Efficiency Analysis, Proceedings in Business and Economics. Springer International Publishing, Switzerland
Balk BM (2018a) Aggregate Productivity and Productivity of the Aggregate: Connecting the Bottom-Up and Top-Down Approaches. In: Greene WH, Khalaf L, Makdissi P, Sickles RC, Veall M, Voia M-C (eds) Productivity and Inequality, Proceedings in Business and Economics. Springer International Publishing, Switzerland

Balk BM (2018b) Empirical Productivity Indices and Indicators. In: Grifell-Tatjé E, Lovell CAK, Sickles RC (eds) The Oxford Handbook of Productivity Analysis. Oxford University Press, New York. Extended version available at SSRN: http://ssrn. com/abstract $=2776956$

Balk BM, Zofío JL (2018) The Many Decompositions of Total Factor Productivity Change, Report No. ERS-2018-003-LIS, Erasmus Research Institute of Management, Retrievable from http://hdl.ha ndle.net/1765/104721. Available at SSRN: http://ssrn.com/abstra $\mathrm{ct}=3167686$

Basu S, Fernald JG (2002) Aggregate productivity and aggregate technology. Eur Econ Rev 46:963-991

Bollard A, Klenow PJ, Sharma G (2013) India's mysterious manufacturing miracle. Rev Econ Dyn 16:59-85

Calver M (2015) On the relationship between gross output-based TFP growth and value added-based TFP growth: an illustration using data from Australian industries. International Productivity Monitor 29:68-82

Diewert WE (1978) Superlative index numbers and consistency in aggregation. Econometrica 46:883-900

Diewert WE (2015) Reconciling gross output TFP growth with value added TFP growth. International Productivity Monitor 29:60-67

Dumagan JC, Balk BM (2016) Dissecting aggregate output and labour productivity change: A postscript on the role of relative prices. $\mathrm{J}$ Prod Anal 45:117-119

Jorgenson DW (2018) Production and welfare: progress in economic measurement. J Econ Lit 56:867-919

Petrin A, Levinsohn J (2012) Measuring aggregate productivity growth using plant-level data. Rand J Econ 43:705-725

Vancauteren M, Veldhuizen E, Balk BM (2012) Measures of Productivity Change: Which Outcome Do You Want? Paper presented at the 32nd General Conference of the IARIW, Boston MA, 5-11 August 2012 